Received 00th January 20xx, Accepted 00th January 20xx DOI: $10.1039 / x 0 \times x 00000 x$

\title{
Influence of Cellulose Nanocrystal Surface Functionalization on the Bending Response of Cellulose Nanocrystal/Ionic Liquid Soft Actuators
}

\author{
Daniela M. Correia, ${ }^{* a},{ }^{b}$ Erlantz Lizundia, ${ }^{* c, d}$ Liliana C. Fernandes, ${ }^{\mathrm{b}, \mathrm{d}}$ Carlos M. Costa ${ }^{\mathrm{b}}$ and Senentxu \\ Lanceros-Méndez ${ }^{\mathrm{d}, \mathrm{e}}$
}

\begin{abstract}
This work reports the development of renewable cellulose nanocrystal (CNC) and ionic liquid (IL) hybrid materials for bending actuator applications. For this purpose, cellulose nanocrystals with different surface charges (neutral, positive and negative) were prepared and increasing amounts of the IL 2-hydroxy-ethyl-trimethylammonium dihydrogen phosphate ([Ch][DHP]) (10 and 25\% wt.) were incorporated into the CNC hosting matrix. The morphology of the samples was evaluated, proving that both surface charge and IL incorporation do not affect the characteristic layered structure of the CNC. Atomic force microscopy results reveal a sea-island morphology in the hybrid films, where CNC bundles are surrounded by [Ch][DHP]-rich regions. An increase in the electrical conductivity is observed upon IL incorporation into the CNC matrix, regardless of the CNC surface charge. The highest electrical conductivity values are observed for IL/CNC (+) $25 \%$ wt. with an electrical conductivity of $3.18 \times 10^{-5} \pm 2.75 \times 10^{-7}{\mathrm{~S} . \mathrm{cm}^{-1}}$ and IL/CNC (-) $10 \% \mathrm{wt}$. $\left(1.26 \times 10^{-5} \pm 5.92 \times 10^{-6} \mathrm{~S}_{\mathrm{cm}} \mathrm{cm}^{-1}\right)$. The highest bending displacement of $2.1 \mathrm{~mm}$ for and applied voltage of $4.0 \mathrm{Vpp}$ at a frequency of $100 \mathrm{mHz}$ was obtained for the IL/CNC (+) $25 \%$ wt composite, demonstrating the suitability of cellulose to develop soft actuators.
\end{abstract}

\section{Introduction}

Natural polymers are gaining increasing attention as a suitable and interesting approach for applications, including biomedical ,${ }^{1}$ sensors ${ }^{2}$ and actuators. ${ }^{3}$ Among the different types of natural polymers, cellulose has been extensively studied due to its availability, processability and tailorable physical-chemical properties. ${ }^{4}$ Cellulose is the most abundant natural polymer on the earth and it is a linear polysaccharide composed by $D$ glucose units bonded through $\beta-1 \rightarrow 4$ glycosidic bonds, and it is obtained by plants and synthesized by a large number of microorganisms such as bacteria. ${ }^{4} 5$ Its chain arrangement presents a fibrillar structure as bundles of microfibrils making the cellulose a high strength biomaterial with superior mechanical properties. 4, 6, 7 Among cellulosic derivatives, cellulose nanocrystals (CNCs) emerge as a suitable platform for the development of multifunctional materials given their nanoscale size, tailored surface functionality, lightweight, mechanical properties and renewability. ${ }^{8}$

\footnotetext{
a. Centre of Chemistry, University of Trás-os-Montes e Alto Douro, 5000-801 Vila Real, Portugal.

b. Centre of Physics, University of Minho, 4710-058 Braga, Portugal. Email: d.correia@fisica.uminho.pt

c. Department of Graphic Design and Engineering Projects, Bilbao Faculty of Engineering, University of the Basque Country (UPV/EHU), Bilbao 48013, Spain. Email: erlantz.liizundia@ehu.eus

d. BCMaterials, Basque Centre for Materials, Applications and Nanostructures, UPV/EHU Science Park, 48940 Leioa, Spain

e. IKERBASQUE, Basque Foundation for Science, 48009 Bilbao, Spain
}

It has been reported that when combined with ionic liquids (ILs) cellulose shows a strong potential for the development of electromechanical soft bending actuators, able to convert electrical signals into a mechanical deformation. ${ }^{8,9}$ Contrarily to electronic electromechanical actuators, the actuator performance is governed by the ions mobility and diffusion into the polymer matrix upon an applied voltage, leading to a mechanical deformation. In this field, significant efforts need to be performed in order to develop sustainable material based actuators working at low voltages with improved speed responses and forces. ${ }^{9}$

ILs are defined as salts composed by organic cations and a variety of organic/inorganic anions and with melting points below $100{ }^{\circ} \mathrm{C} .{ }^{10}$ They show low vapor pressure, electrochemical and chemical stability, and are typically liquid at room temperature. Other interesting properties attributed to ILs are their high miscibility and solubility with a wide variety of compounds and their high ionic conductivity, in the order of $\sim 10^{-3}-10^{-2} \mathrm{~S} \cdot \mathrm{cm}^{-1} .{ }^{10}$ The incorporation of ILs into a polymer matrix allows the development of ionic polymer-based composites suitable for the development of sensors and actuators with low weight, fracture tolerance and mechanical flexibility.

ILs have been combined with different polymers for the development of electromechanical actuators. Those polymers include poly(vinylidene fluoride) (PVDF) and its co-polymers, ${ }^{11-}$ ${ }^{13}$ poly(L-lactic acid) (PLLA), ${ }^{14}$ poly ( $\varepsilon$-caprolactone) (PCL), ${ }^{15}$ and poly(ethylene oxide) (PEO). ${ }^{16}$ However, besides the high interest devoted on ILs, few studies report on the development of naturally derived polymer based electromechanical 
actuators, and particularly cellulose/IL composites for actuators. 17

Actuators based on carbon nanotubes covalently grafted with cellulose, nanocoated with a conductive polymer polypyrrole (PPy) and ionic liquids exhibited durable bending actuation at ambient humidity and temperature condition. ${ }^{18}$ Carboxylated bacterial cellulose (CBC), 1-ethyl-3-methylimidazolium tetrafluoroborate $\left([\mathrm{EMIM}]\left[\mathrm{BF}_{4}\right]\right) \quad \mathrm{IL}$, and poly $(3,4-$ ethylenedioxythiophene)-poly(styrenesulfonate) (PEDOT:PSS) electrodes were also evaluated as electromechanical actuators, showing a displacement of $4.9 \mathrm{~mm}$ under an applied voltage of 2.5 V. ${ }^{19}$ A soft actuator composed by highly conductive polyaniline (PANI) doped with reduced graphene oxide/carbon nano-sheets and cellulose-based electrolyte dissolved in the ionic liquid 1-butyl-3-methylimidazolium chloride ([Bmim][Cl]) revealed a large static deformation (up to $13.53 \mathrm{~mm}$ ) and bending force (up to $12 \mathrm{mN}$ ) at $5.0 \mathrm{~V} \mathrm{DC} .{ }^{17}$ The IL 2-hydroxyethyl-trimethylammonium dihydrogen phosphate ([Ch][DHP]) has been also incorporated into cellulose nanocrystals and in water-soluble methyl cellulose (MC), hydroxypropyl cellulose (HPC), and sodium carboxymethyl cellulose ( $\mathrm{NaCMC}$ )-cellulose derivatives, for the development of soft actuators, the composite with the larger bending displacement of $9 \mathrm{~mm}$ at 8.0 Vpp being $\mathrm{NaCMC} /[\mathrm{Ch}][\mathrm{DHP}] .{ }^{8}$ Despite having already studied the influence of the IL content and cellulose derivative type on the physical-chemical properties and bending response of the resulting materials, another important aspect will be the study of how CNC surface charge (neutral, positive and negative) affects the interaction with IL and thus, the bending response. In this context, the present work reports on IL/ CNC composites for bending actuator applications. In particular, CNCs have been selected as they can be prepared with different surface charges (neutral, positive and negative) in order to tune their interaction with the IL and to tailor the physical-chemical properties of the hybrid materials and the corresponding bending actuator response. In this sense, this work will contribute for the improvement of naturally derived polymers in the field of actuators applications, through the understanding of the physical-chemical interactions between a charged biopolymer and the IL [Ch][DHP].

\section{Experimental}

\section{Materials}

Microcrystalline cellulose with an average particle size of $20 \mu \mathrm{m}$ (310697-500G), sulphuric acid (95-97\%, 30743-1L), sodium hydroxide pellets (97\%, Sigma-Aldrich) and glycidyltrimethylammonium chloride (50053-50ML) were supplied by Sigma Aldrich. The IL 2-hydroxyethyltrimethylammonium dihydrogen phosphate ([Ch][DHP]) (purity higher than $>98 \%$ ) [Ch] [DHP] was acquired from lolitec.

\section{Sample preparation}

Cellulose nanocrystals (CNCs) were extracted from microcrystalline cellulose through sulphuric-acid hydrolysis followed by tip-sonication as previously reported. ${ }^{20}$ An aqueous dispersion of $\mathrm{CNCs}(\mathrm{pH} 1.9$, concentration $1.5 \% \mathrm{w} / \mathrm{w})$ with surface anionic sulfate ester groups $\left(-\mathrm{OSO}_{3}{ }^{-}\right)$is obtained through this process. Slightly negative CNCs were obtained upon treating water-dispersed CNCs with a $2 \mathrm{M} \mathrm{NaOH}$ solution at $60 \stackrel{\circ}{ }{ }^{20}$. After $2.5 \mathrm{~h}$, the reaction was quenched with 5 -fold distilled water and the excess of $\mathrm{NaOH}$ was removed by centrifugation. Positive groups onto CNCs were incorporated after the reaction of CNCs with glycidyltrimethylammonium chloride to yield CNCs with cationic hydroxypropyltrimethylammonium chloride substituents (Scheme 1). Water dispersed CNCs were exchanged into a $7 \%$ $\mathrm{w} / \mathrm{v} \mathrm{NaOH}$ solution. After 30 minutes at room temperature, 10 $\mathrm{mg}$ of EPTMAC were added and it was allowed to react for $5 \mathrm{~h}$ at 65 ㅇ․ ${ }^{21}$ The reaction was stopped by adding 5-fold distilled water followed by centrifugation.

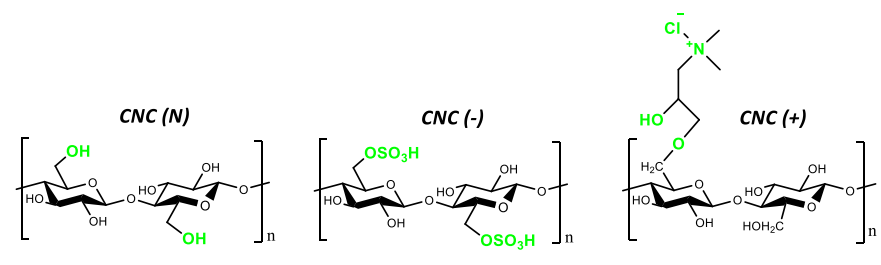

Scheme 1. Chemical structure of the original and functionalized CNCs.

Films based on modified CNCs and 2-hydroxyethyltrimethylammonium dihydrogen phosphate [Ch][DHP] were prepared by the solvent evaporation method as previously described. ${ }^{8}$ Different contents of [Ch][DHP] (0, 10 and $25 \%$ wt.) were added to an aqueous solution containing $\mathrm{CNC}$ and mixed at room temperature under magnetic stirring. After dissolution, solutions were casted in Petri-dishes and dried at room temperature for $72 \mathrm{~h}$. Samples with a typical thickness of $56 \pm$ $14 \mu \mathrm{m}$ were obtained.

\section{Sample characterization}

The extent of CNC surface modification was characterized by zeta potential and attenuated total reflectance Fourier transform infrared spectroscopy (ATR-FTIR) measurements. Zeta potential of water-dispersed CNCs $\left(0.1 \mathrm{mg} \cdot \mathrm{mL}^{-1}\right)$ was measured using a Malvern Zetasizer Nano-ZS. ATR-FTIR spectra were recorded in a Jasco FT/IR-4100 system with a resolution of $2 \mathrm{~cm}^{-1}$. TGA was performed in a Mettler Toledo TGA/SDTA 851e instrument under a $\mathrm{N}_{2}$ atmosphere $\left(50 \mathrm{~mL} \cdot \mathrm{min}^{-1}\right)$ at a heating rate of $10^{\circ} \mathrm{C} \cdot \mathrm{min}^{-1}$

Fractured film surfaces were coated with gold-palladium by magnetron sputtering (Polaron SC502) and their morphology was evaluated by field-emission scanning electron microscope (FESEM) in a HitachiS-4800 at an acceleration voltage of $5 \mathrm{kV}$.

The surface features of IL/CNC films were analyzed using a Dimension ICON scanning probe microscope (SPM) from Bruker. Experiments were performed in tapping mode using an integrated silicon tip/cantilever.

X-ray powder diffraction (XRD) patterns were measured using a Bruker D8 Advance diffractometer equipped with a Cu tube, Ge (111) incident beam monochromator ( $\lambda=1.5406 \AA$ A) (fixed slit 1 $\mathrm{mm}$ ) and a Sol-X energy dispersive detector (fixed slit $0.06 \mathrm{~mm}$ ). 
The samples were mounted on a zero-background silicon wafer embedded in a generic sample holder.

The volume DC electrical conductivity of the films was calculated after measuring the characteristic current-voltage (IV) curves. The measurements were performed at room temperature upon the application of voltages ranging from -10 to $10 \mathrm{~V}$ using an automated Keithley 487 picoammeter/voltage source. Previously to the measurements, circular gold electrodes ( $5 \mathrm{~mm}$ diameter) were coated on both sides of the samples using a magnetron sputtering (Scancoat Six SEM Sputter Coater). The electrical conductivity of the samples was evaluated from the $I-V$ slope taking into consideration their geometrical characteristics. 8,11

The bending actuator response was evaluated by electromechanical tests, as described in. ${ }^{22,} 23$ Samples with dimensions of $12 \mathrm{~mm} \times 2 \mathrm{~mm}$ were first gold coated onto both sides by magnetron sputtering (Scancoat Six SEM Sputter Coater). Then, the samples were placed in a home-made sample holder, connected to an Agilent 33220A function generator and to an oscilloscope PicoScope 4227, and the bending displacement was recorded with a high-definition camera Logitech HD Webcam C615and evaluated at room temperature using a square wave signal at an applied voltage of $4 \mathrm{Vpp}$ at a frequency of $100 \mathrm{mHz}$.

\section{Results and discussion}

\section{CNC modification}

Originally, CNCs have been synthesized after a sulfuric acid hydrolysis process, resulting in negatively charged nanoparticles (zeta-potential of $-45.2 \pm 1.1 \mathrm{mV}$ ) due to the anionic sulfate half-ester groups $\left(-\mathrm{OSO}_{3}{ }^{-}\right)$onto $\mathrm{CNC}$ surfaces. ${ }^{24}$ The numerous hydroxyl groups onto CNCs allow introducing a tailored surface charge by simple chemical treatments. A $2 \mathrm{M}$ $\mathrm{NaOH}$ treatment at $60{ }^{\circ} \mathrm{C}$ removes the $-\mathrm{OSO}_{3}$ - groups, rendering CNCs with a low zeta-potential of $-4.1 \pm 0.3 \mathrm{mV}$. We also turned negatively-charged CNCs into positively-charged CNCs (zetapotential of $+21.4 \pm 0.7 \mathrm{mV}$ ) through a cationization reaction involving epoxypropyltrimethylammonium chloride. As shown in Fig. S1a), surface functionalization of CNC can be followed by Fourier transform infrared spectroscopy. Indeed, CNC desulfation results in a notable intensity decrease of the stretching vibration located at $1033 \mathrm{~cm}^{-1}$ corresponding to the $-\mathrm{SO}_{3}$ groups, while the intensity arising from the $\mathrm{O}-\mathrm{H}$ stretching vibration region of cellulose is increased (Fig. S1b). The occurrence of positively charged CNCs is further proved by the increased intensity of the band at $1640 \mathrm{~cm}^{-1}$ (see Fig. S1c), which indicates a cationization-induced hydrophilization due to the adsorbed water. Moreover, upon removal of the sulfate groups, modified CNCs present an enhanced thermal stability due to the inhibition of the dehydration catalyst effect of $-\mathrm{OSO}_{3}$ groups, which is manifested into an increased onset of thermal degradation (according to the first $10 \%$ weight loss) by nearly $55^{\circ} \mathrm{C}$ from the $184.3^{\circ} \mathrm{C}$ of the original negatively-charged CNCs (see Fig. S1d). ${ }^{25}$

\section{Morphological characterization}

As summarized in Fig. 1, composite morphology was first assessed by analyzing the cryo-fractured cross-section images obtained by scanning electron microscopy (SEM). Films composed solely by CNCs (neat CNC) present a rough layered structure which is obtained after the evaporation induced selfassembly (EISA) process, where individual CNCs assemble together to form a free-standing film. ${ }^{26-28}$. This characteristic morphology is not perturbed upon CNC charge modification or the introduction of [Ch][DHP] (Fig. 1), indicating that such IL is compatible with the self-assembly process of CNCs.

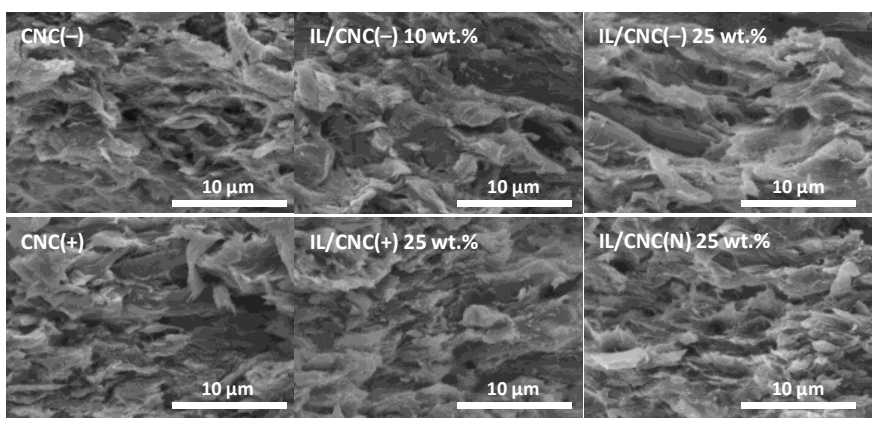

Fig. 1. SEM images showing the cross-section of surface charge modified CNC and the corresponding IL/CNC films with different IL contents.

Atomic force microscopy (AFM) has been performed to get further insights on the structure of surface charge modified CNC films and IL/CNC composite films. Fig. 2 shows the tappingmode SPM amplitude images $\left(5 \times 5 \mu \mathrm{m}^{2}\right)$ of pure cellulosic films composed solely by negative and positive CNCs (CNC - and CNC + respectively) and the corresponding 10 and $25 \mathrm{wt} . \% \mathrm{IL}$ composites. A homogeneously distributed CNC network is observed for all samples, where CNCs remain parallel to the surface as a consequence of the EISA process, where upon water evaporation, the surface interaction of CNCs forces them to adopt a parallel arrangement. ${ }^{29}$ The hybrid films show a seaisland morphology, where CNC bundles are surrounded by [Ch][DHP]-rich regions. Although not fully well-dispersed, the relatively small size of such aggregated regions of approximately $600 \mathrm{~nm}$ yields macroscopically homogeneous films, independently of the CNC surface charge and the IL content. 


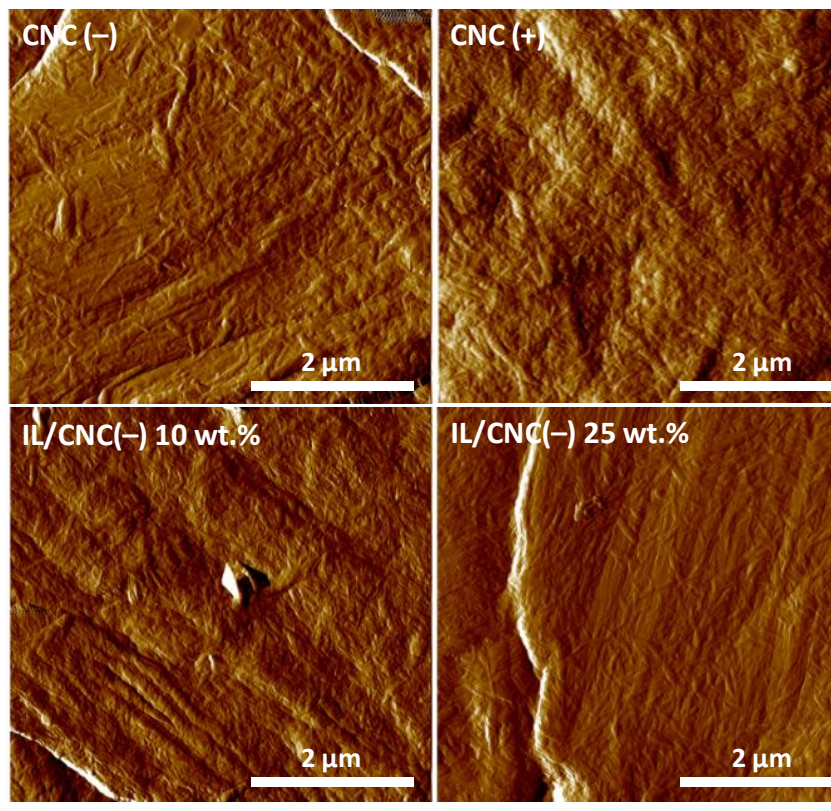

Fig. 2. Tapping-mode SPM amplitude images of surface charge modified CNC films and representative IL/CNC films with different IL content.

\section{Crystalline structure}

X-ray diffraction (XRD) patterns of the films are shown in Fig. $3 a$. Independently of their surface charge, all CNC films present several diffraction maxima at $2 \vartheta=14.9,16.5,20.5,22.7$ and $34.4^{\circ}$ which arise from the (1-10), (101), (102), (200) and (004) planes of cellulose $I \beta,{ }^{30}, 31$ suggesting that surface modification does not affect the original crystalline structure of CNCs. Apart from small intensity changes, no significant modifications on the diffraction patters (either the presence of new peaks or the shifting/extinction of existing ones) are observed upon [Ch][DHP] incorporation. These results indicate that composite films are composed by crystalline CNC together with amorphous ionic liquid regions, where no long-range order chlorine structures are formed. 32 This predominantly amorphous structure surrounded by CNCs is consistent with the morphological observations obtained by AFM and is suitable towards the development of bending actuators, where materials with significant amorphous content are preferred, allowing higher ionic mobility and bendability. 33
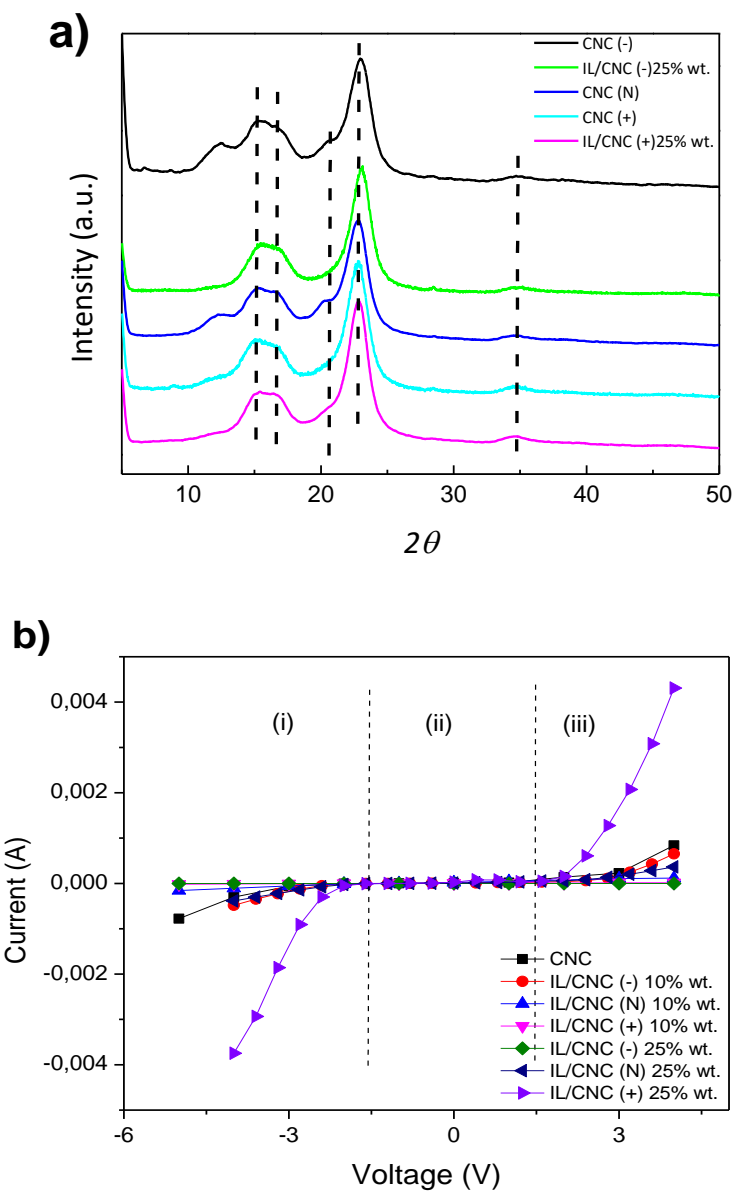

Fig. 3. a) XRD patterns of IL/CNC films. b) I-V curves of CNC and IL/CNC films comprising different IL contents and CNC surface charges.

\section{Electrical properties}

The effect of CNC surface charge modification and IL content into the electrical properties of the IL/CNC samples was evaluated from the current vs voltage (I-V) curves presented in Fig. 3b. Neat CNC presents a non-linear regime composed by two main regions, one between -2.0 and $+2.0 \mathrm{~V}$ (ii) and another for higher voltage (iii), as reported in. ${ }^{8} \mathrm{As}$ it is shown in Fig. $3 \mathrm{~b}$, with the exception of the IL/CNC (+) $10 \%$ wt. and IL/CNC (-) $25 \%$ wt., this behavior is also observed for the IL/CNC samples, being the two stages more pronounced for the IL/CNC (-) 10\% wt. and IL/CNC (+) $25 \%$ wt. For the latter, a first step for an applied voltage lower than $-2 \mathrm{~V}$ occurs (i). For the other samples comprising different IL/CNC contents and CNC charges, the I-V behavior is characterized by a quasilinear regime. The observed different slopes upon the IL incorporation results from the IL cations and anions mobility within the CNC matrix. As a result of the strong interactions that occurs between the IL and CNC matrix, the mobility is favored for an applied voltage higher than $2.0 \mathrm{~V}$, the applied field allowing to overcome the IL-polymer electrostatic interaction. ${ }^{8}$ Additionally, those results indicate that in the IL/CNC (+) $25 \%$ wt. sample the charge-transfer is facilitated in the presence of positively charges CNCs, ${ }^{34}$ where 
the IL can adsorb positively charged moieties originating from the alkali-activated cellulose $-\mathrm{OH}$ to the epoxy moiety of EPTMAC. The effect of the IL content and CNC charge in the electrical conductivity of the CNC and IL/CNC samples was evaluated from the slope of I-V curves ${ }^{14}$ presented in Fig. $3 \mathrm{~b}$

Table 1. DC electrical conductivity of the CNC and IL/CNC comprising different IL contents and CNC charges.

\begin{tabular}{c|c|c|c}
\hline \multicolumn{4}{c}{ Electrical conductivity $\left(\mathbf{S ~ c m}^{-1}\right)$} \\
\hline Sample & (i) & (ii) & $($ iii) \\
\hline CNC & $1.02 \times 10^{-9} \pm$ & $3.82 \times 10^{-11} \pm$ & $1.40 \times 10^{-9} \pm$ \\
& 0.0 & 0.0 & 0.0 \\
\hline CNC 10\% & $1.08 \times 10^{-5} \pm$ & $5.67 \times 10^{-7} \pm$ & $1.26 \times 10^{-5} \pm$ \\
negative & $4.07 \times 10^{-7}$ & $8.00 \times 10^{-8}$ & $5.92 \times 10^{-6}$ \\
\hline CNC 10\% & $1.79 \times 10^{-6} \pm$ & $6.7 \times 10^{-7} \pm$ & $1.76 \times 10^{-6} \pm$ \\
neutral & $3.77 \times 10^{-7}$ & $2.74 \times 10^{-8}$ & $3.81 \times 10^{-7}$ \\
\hline CNC 10\% & $1.27 \times 10^{-7} \pm$ & $5.85 \times 10^{-8} \pm$ & $9.57 \times 10^{-8} \pm$ \\
positive & $9.44 \times 10^{-10}$ & $2.63 \times 10^{-9}$ & $1.58 \times 10^{-9}$ \\
\hline CNC 25\% & $5.25 \times 10^{-12} \pm$ & $2.88 \times 10^{-12} \pm$ & $1.55 \times 10^{-12} \pm$ \\
negative & $1.91 \times 10^{-13}$ & $3.8 \times 10^{-13}$ & $1.23 \times 10^{-13}$ \\
\hline CNC 25\% & $4.85 \times 10^{-6} \pm$ & $3.45 \times 10^{-7} \pm$ & $3.76 \times 10^{-6} \pm$ \\
neutral & $2.74 \times 10^{-7}$ & $2.15 \times 10^{-8}$ & $1.95 \times 10^{-7}$ \\
\hline CNC 25\% & $3.31 \times 10^{-5} \pm$ & $8.02 \times 10^{-7} \pm$ & $3.18 \times 10^{-5} \pm$ \\
positive & $3.34 \times 10^{-6}$ & $4.54 \times 10^{-8}$ & $2.75 \times 10^{-7}$ \\
\hline
\end{tabular}

From Table 1 it is observed an increase in the DC electrical conductivity of the IL/CNC samples upon the IL incorporation into the CNC matrix, being independent of the CNC charge modification. It is also to notice that the electrical conductivity of IL/CNC samples increases with increasing IL content but that this is a complex process due to the variety of interactions existing in the hybrid materials - anions and cations in the IL and varying surface charge of the CNC. Thus, not a specific trend is observed related to the surface charge type of the CNC or the IL content. The increase in the electrical conductivity value occurs in all three regimes, being not observed significant differences between the regimes $\mathrm{i}$ and ii with the IL content increase and CNC charge. The highest DC electrical conductivity values are observed in regime iii, indicating a higher number of ionic charges (cations and anions) carriers and ions mobility into the CNC matrix for this regime upon an applied voltage. The highest conductivity values are observed for IL/CNC (+) $25 \%$ wt. with an electrical conductivity of $3.18 \times 10^{-5} \pm 2.75 \times 10^{-7}{\mathrm{~S} . \mathrm{cm}^{-1}}^{-1}$ and IL/CNC (-) $10 \%$ wt. $\left(1.26 \times 10^{-5} \pm 5.92 \times 10^{-6}{\left.\mathrm{~S} . \mathrm{cm}^{-1}\right)}^{-1}\right.$ (regime iii). With respect to the possible interaction mechanisms, the lowest electrical conductivity has been obtained for the films containing neutral CNCs because of the lower ability of the choline cation (within the IL) to interact with CNCs decorated solely by $-\mathrm{OH}$ (hydroxyl groups). On the contrary, the samples containing positively-charged CNCs display the largest electrical conductivity, which can be explained as follows: CNC cationization via reaction of the epoxy moiety of EPTMAC with alkali-activated CNC hydroxyl groups result in CNCs decorated with hydroxypropyltrimethylammonium chloride substituents. Upon [Ch][DHP] IL incorporation, the chloride counter anion on the CNC surface (see Scheme 2) interacts with the choline cation within the IL, ${ }^{35,} 36$ facilitating the formation of additional charge carriers which enhance the overall conductivity. The lower electrical conductivity values for the IL/CNC (-) samples when compared to the IL/CNC (+) samples comprising $25 \%$ wt. of IL indicates that the effect of increased number of charge carriers, leading to increasing electrical conductivity, is overcome by the electrostatic repulsive and attractive interaction between the large number of anions and cations, reducing ion mobility and electrical conductivity for the samples with larger IL content. It is to notice that lower IL contents favour the interaction between the cations with the negatively CNC charge as shown from the conductivity values observed in the sample IL/CNC (+) $10 \% \mathrm{wt}$

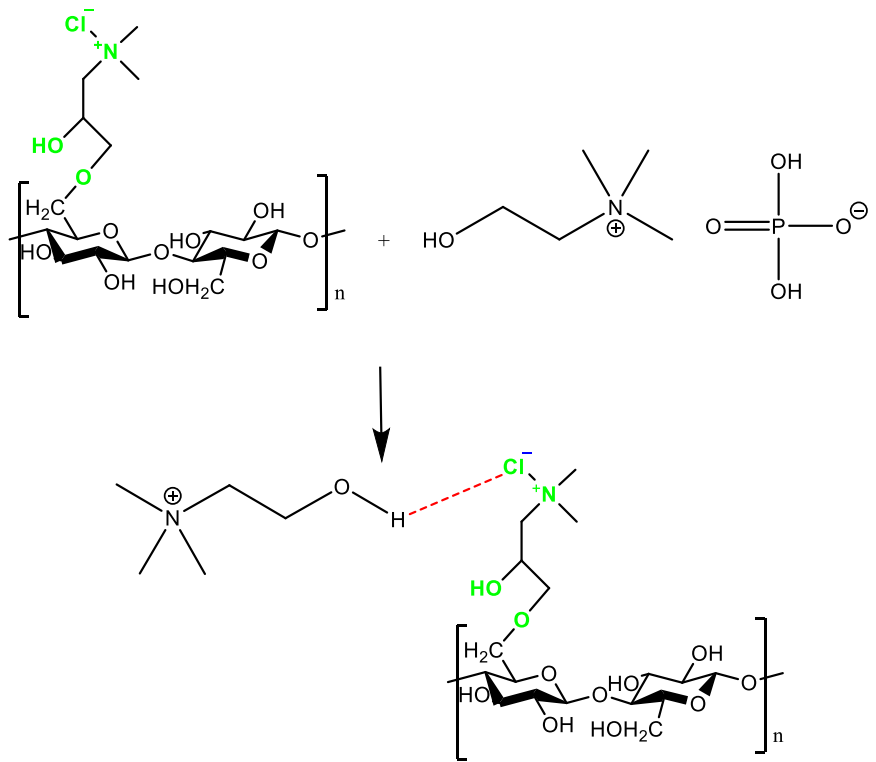

Scheme 2. Proposed mechanism of $\mathrm{CNC}(+)$ interaction with the $[\mathrm{Ch}]^{+}$anion.

\section{Bending actuation performance}

Upon self-assembly, CNCs can form free-standing materials, enabling the fabrication of actuator films and membranes. ${ }^{37}$ The actuator performance of the developed IL/CNC composites comprising CNC with different surface charges with 25 wt\% of IL was evaluated by measuring the maximum displacement from the distance of the tip of the actuator from the relaxed position up to the maximum displacement upon an applied voltage of $4.0 \mathrm{Vpp}$, once these composites present the highest electrical conductivity value.

It is to notice that just the samples IL/CNC (N) and IL/CNC (+) comprising $25 \% \mathrm{wt}$. developed a displacement as a function of the applied voltage (Fig. 4a). The absence of bending response in the IL/CNC (-) samples resulted from the lower electrical conductivity values for the samples containing the higher IL amount and to the hinderance of IL (cation)-CNC negative surface charge interaction. Upon an applied voltage, the cations and anions tend to move and diffuse into the cellulose matrix to the positive (anions) and negative (cations) side of the electrode layers, resulting in the accumulation of the ions close to the electrodes. ${ }^{3,12}$ This trend results in the observed displacement motion. Further, higher IL contents promotes a decrease in the 
Young's modulus due to the IL plasticizing effect as a result of the IL penetration between adjacent $\mathrm{CNCs}$, reducing their hydrogen bonding-induced entanglement (see Fig. S2). ${ }^{8}$

According to the optical photographs in Fig. $4 \mathrm{~b}$, the composite IL/CNC (N) $25 \%$ wt. shows a displacement of $1.1 \mathrm{~mm}$ and the composite IL/CNC (+) $25 \%$ wt. a displacement of $2.1 \mathrm{~mm}$ for an applied voltage of $4.0 \mathrm{Vpp}$, respectively. Similarly, results were obtained in. ${ }^{8}$ Therefore, the positive outcome regarding the conductivity enhancement in the presence of positively charged CNCs is translated into a larger displacement for a given voltage application, enabling the fabrication of bending actuators. Those results further indicate that the cations and anions of the IL interact preferentially with the positive charge on the surface of CNCs.

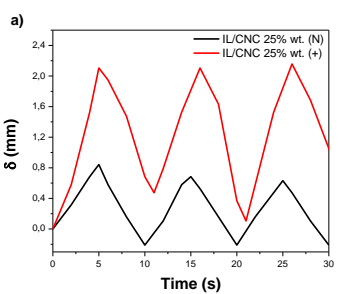

b)
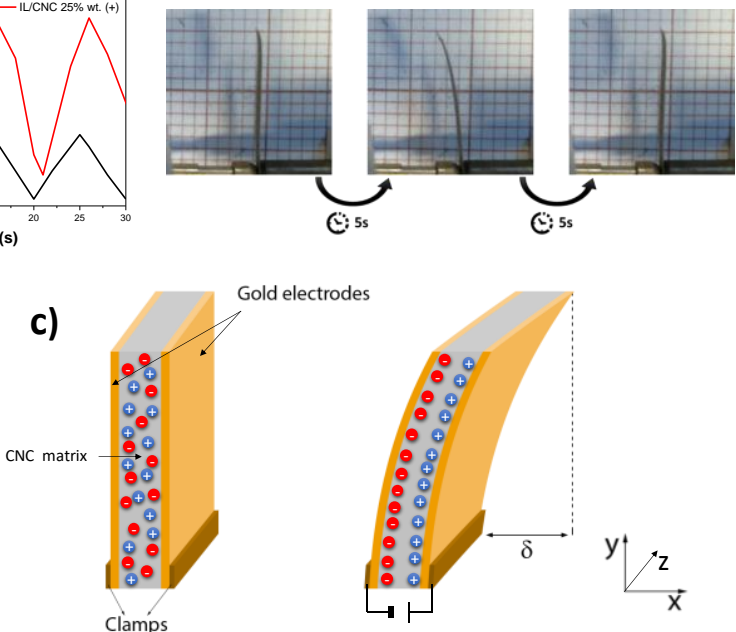

Fig. 4. Displacement of the composites for: (a) IL/CNC (N) $25 \%$ wt. and IL/CNC (+ $25 \%$ wt. for an applied voltage of $4 \mathrm{Vpp}$ at a frequency of $100 \mathrm{mHz}$. b) Bending schematic representation of the cations and anions movement from the IL upon and applied voltage.

In fact, the introduction of IL into a neutral CNC matrix induces a bending motion resulting from the cations and anions movement in response to an applied electrical field to the negative and positive side of the electrodes (see the schematic representation in Fig. $4 \mathrm{c}$ ). ${ }^{8}$ Attending to the obtained bending results, the bending motion depends both on IL content and CNC surface charge. Among all samples, IL/CNC (+) develop the largest bending response upon applied voltages of $4.0 \mathrm{Vpp}$, showing that CNC positive surface modification favours the higher IL charge separation and movement across the polymer matrix, resulting in a larger bending motion. From Fig. 4 it is also noticeable the asymmetric displacement of both samples over time showing that no cross-over of ionic species occur, which will lead to symmetric bending. The performance stability of the sample is demonstrated by the similar actuator performance after 1 month (see Fig. S3). Thus, even with lower actuator displacements when compared with other IL/polymer matrixes, ${ }^{11,17,38}$ the obtained results demonstrate the suitability of this naturally-derived material to be applied as soft actuators.

\section{Conclusions}

Cellulose nanocrystals have been used for the development of bending actuators. [Ch][DHP] IL/CNC composites comprising different CNC surface charges (neutral, positive and negative) and IL contents were obtained following a solvent casting method. Independently of the CNC surface charge and IL content, no significant differences were observed in the rough layered structure of the CNC. A sea-island morphology where the CNC bundles remain surrounded by [Ch][DHP]-rich regions was observed by AFM. Similarly, the XRD patterns of the samples revealed no differences between neat CNC and IL/CNC composites, where the composites are constituted by crystalline CNC together with amorphous ionic liquid regions. The electrical measurements performed from current-voltage curves showed two electrical conductivity regimes for applied voltages larger than $+/-2 \mathrm{~V}$. IL/CNC (+) $25 \% \mathrm{wt}$. developed the highest conductivity value $\left(3.18 \times 10^{-5} \pm 2.75 \times 10^{-7} \mathrm{~S} \cdot \mathrm{cm}^{-1}\right)$, followed by the IL/CNC (-) $10 \%$ wt. $\left(1.26 \times 10^{-5} \pm 5.92 \times 10^{-6}\right.$ $\left.\mathrm{S} \cdot \mathrm{cm}^{-1}\right)$. The highest conductivity values observed for the IL/CNC (+) 25 wt.\% resulted from the chloride counter anion interaction with the choline cation from the IL.

Finally, the response of the films upon an applied voltage revealed a high displacement observed for the composite IL/CNC (+) $25 \%$ wt. ( $2.1 \mathrm{~mm}$ ) for an applied voltage of $4.0 \mathrm{Vpp}$, respectively, at a frequency of $100 \mathrm{mHz}$. Thus, bending actuators based on renewable natural materials can be developed and tailored for applications based on polymer-IL charge interactions.

\section{Author Contributions}

Daniela M. Correia: Methodology; Validation; Formal analysis; Investigation; Writing - Original Draft; Writing - Review \& Editing

Erlantz Lizundia: Methodology; Validation; Formal analysis; Investigation; Writing - Original Draft; Writing - Review \& Editing.

Liliana C. Fernandes: Investigation; Validation; Writing - Original Draft

Carlos M. Costa: Methodology; Validation; Formal analysis; Investigation; Writing - Original Draft; Writing - Review \& Editing

Senentxu Lanceros-Mendez: Methodology; Resources; Funding acquisition; Conceptualization; Validation; Project administration; Writing - Review \& Editing

\section{Conflicts of interest}

There are no conflicts to declare.

\section{Acknowledgements}

The authors thank FCT - Fundação para a Ciência e Tecnologia for financial support under the framework of the Strategic 
Funding UID/FIS/04650/2019 and UID/QUI/50006/2019 and projects PTDC/BTM-MAT/28237/2017, PTDC/EMDEMD/28159/2017 and PTDC/FIS-MAC/28157/2017. D.M.C., C.M.C and L.C.F. also acknowledge to FCT by the grants SFRH/BPD/121526/2016, SFRH/BPD/112547/2015 and $\mathrm{SFRH} / \mathrm{BD} / 145345 / 2019$, respectively. The authors thank funding by the Spanish State Research Agency (AEI) and the European Regional Development Fund (ERFD) through the project PID2019-106099RB-C43 / AEI / $10.13039 / 501100011033$ and from the Basque Government Industry and Education Department under the ELKARTEK and PIBA (PIBA-2018-06) programs, respectively. SGlker (UPV/EHU, MICINN, GV/EJ, ERDF and ESF) support is gratefully acknowledged.

\section{Notes and references}

1 B. ter Horst, N. S. Moiemen and L. M. Grover, in Biomaterials for Skin Repair and Regeneration, ed. E. García-Gareta, Woodhead Publishing, 2019, DOI: https://doi.org/10.1016/B978-0-08-102546-8.00006-6, pp. 151-192.

2 A. Reizabal, S. Gonçalves, R. Brito-Pereira, P. Costa, C. M. Costa, L. Pérez-Álvarez, J. L. Vilas-Vilela and S. LancerosMéndez, Nanoscale Advances, 2019, 1, 2284-2292.

3 A. Reizabal, D. M. Correia, C. M. Costa, L. Perez-Alvarez, J. L. Vilas-Vilela and S. Lanceros-Méndez, ACS Applied Materials \& Interfaces, 2019, 11, 30197-30206.

4 C. Brigham, in Green Chemistry, eds. B. Török and T. Dransfield, Elsevier, 2018, DOI: https://doi.org/10.1016/B978-0-12-809270-5.00027-3, pp 753-770.

5 L. Geng, N. Mittal, C. Zhan, F. Ansari, P. R. Sharma, X. Peng, B. S. Hsiao and L. D. Söderberg, Macromolecules, 2018, 51, 14981506.

6 K. Okuda and S. Sekida, in Progress in Biotechnology, eds. N. Morohoshi and A. Komamine, Elsevier, 2001, vol. 18, pp. 93 100.

7 Y. Li, J. Wang, X. Liu and S. Zhang, Chemical Science, 2018, 9, 4027-4043.

8 D. M. Correia, E. Lizundia, R. M. Meira, M. Rincon-Iglesias and S. Lanceros-Mendez, Materials, 2020, 13.

9 B. T. White and T. E. Long, Macromolecular Rapid Communications, 2019, 40, 1800521.

10 D. M. Correia, L. C. Fernandes, P. M. Martins, C. GarciaAstrain, C. M. Costa, J. Reguera and S. Lanceros-Mendez, Advanced Functional Materials, 2020, 30.

11 D. M. Correia, J. C. Barbosa, C. M. Costa, P. M. Reis, J. M. S. S. Esperança, V. de Zea Bermudez and S. Lanceros-Méndez, The Journal of Physical Chemistry C, 2019, 123, 12744-12752.

12 R. Mejri, J. C. Dias, S. Besbes Hentati, G. Botelho, J. M. S. S. Esperança, C. M. Costa and S. Lanceros- Mendez, European Polymer Journal, 2016, 85, 445-451.

13 K. Asaka, Synthesiology, 2016, 9, 117-123.

14 D. M. Correia, L. C. Fernandes, B. D. D. Cruz, G. Botelho, V. d. Z. Bermudez and S. Lanceros-Mendez, Polymers, 2020, 12.

15 Y. Ren, Z. Zhou, G. Yin, G.-X. Chen and Q. Li, RSC Advances, 2016, 6, 31351-31358.

16 K. Elamin, M. Shojaatalhosseini, O. Danyliv, A. Martinelli and J. Swenson, Electrochimica Acta, 2019, 299, 979-986.

17 P. Martins, D. M. Correia, V. Correia and S. Lanceros-Mendez, Physical Chemistry Chemical Physics, 2020, 22, 15163-15182.

18 J. Kim, S. Yun, S. K. Mahadeva, K. Yun, S. Y. Yang and M. Maniruzzaman, Sensors, 2010, 10, 1473-1485.
19 F. Wang, J. H. Jeon, S. Park, C. D. Kee, S. J. Kim and I. K. Oh, Soft Matter, 2016, 12, 246-254.

20 E. Lizundia, T.-D. Nguyen, Jose L. Vilas, W. Y. Hamad and M. J. MacLachlan, Journal of Materials Chemistry A, 2017, 5, 1918419194

21 M. Hasani, E. D. Cranston, G. Westman and D. G. Gray, Soft Matter, 2008, 4, 2238-2244.

22 R. Mejri, J. C. Dias, S. B. Hentati, M. S. Martins, C. M. Costa and S. Lanceros-Mendez, Journal of Non-Crystalline Solids, 2016, 453, 8-15.

23 J. C. Dias, D. M. Correia, C. M. Costa, C. Ribeiro, A. Maceiras, J. L. Vilas, G. Botelho, V. de Zea Bermudez and S. LancerosMendez, Electrochimica Acta, 2019, 296, 598-607.

24 E. Lizundia, D. Puglia, T.-D. Nguyen and I. Armentano, Progress in Materials Science, 2020, 112, 100668.

25 N. Lin and A. Dufresne, Nanoscale, 2014, 6, 5384-5393.

26 A. Tran, W. Y. Hamad and M. J. MacLachlan, Langmuir, 2018, 34, 646-652.

27 T.-D. Nguyen, E. Sierra, H. Eguiraun and E. Lizundia, European Journal of Physics, 2018, 39, 045803.

28 A. G. Dumanli, H. M. van der Kooij, G. Kamita, E. Reisner, J. J. Baumberg, U. Steiner and S. Vignolini, ACS Applied Materials \& Interfaces, 2014, 6, 12302-12306.

29 M. Giese, L. K. Blusch, M. K. Khan and M. J. MacLachlan, Angewandte Chemie International Edition, 2015, 54, 28882910.

30 P. Lu and Y.-L. Hsieh, Carbohydrate Polymers, 2010, 82, 329336.

31 M. A. Lucchini, E. Lizundia, S. Moser, M. Niederberger and G. Nyström, ACS Applied Materials \& Interfaces, 2018, 10, 29599-29607.

32 P. Barbosa, J. Campos, A. Turygin, V. Y. Shur, A. Kholkin, A. Barros-Timmons and F. M. Figueiredo, Journal of Materials Chemistry C, 2017, 5, 12134-12142.

33 E.-J. Shin, W.-H. Park and S.-Y. Kim, Applied Sciences, 2018, 8, 1284 .

34 O. A. T. Dias, S. Konar, A. L. Leão, W. Yang, J. Tjong and M. Sain, 2020, 8.

35 C. R. Ashworth, R. P. Matthews, T. Welton and P. A. Hunt, Physical Chemistry Chemical Physics, 2016, 18, 18145-18160.

36 A. Pinkert, K. N. Marsh, S. Pang and M. P. Staiger, Chemical Reviews, 2009, 109, 6712-6728.

37 E. Lizundia, M. Jimenez, C. Altorfer, M. Niederberger and W. Caseri, Cellulose, 2019, 26, 5513-5527.

38 D.M. Correia, L.C.Fernandes P.M. Martins C. García-Astrain C.M. Costa J. Reguera and S. Lanceros-Méndez, Advanced Funcitonal Materials, 2020, 30, 1909736. 